\title{
Using Constrained Optimization for the Allocation of COVID-19 Vaccines in the Philippines
}

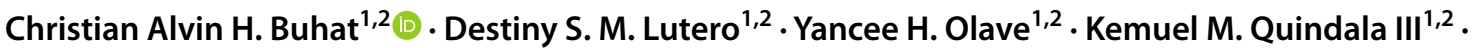 \\ Mary Grace P. Recreo $o^{1,2}$. Dylan Antonio S. J. Talabis ${ }^{1,2}$. Monica C. Torres ${ }^{1,2}$. Jerrold M. Tubay ${ }^{1,2}$. \\ Jomar F. Rabajante ${ }^{1,2}$
}

Accepted: 4 June 2021 / Published online: 25 June 2021

(c) The Author(s), under exclusive licence to Springer Nature Switzerland AG 2021

\begin{abstract}
Background Vaccine allocation is a national concern especially for countries such as the Philippines that have limited resources in acquiring COVID-19 vaccines. As such, certain groups are suggested to be prioritized for vaccination to protect the most vulnerable before vaccinating others.

Objective The study aims to determine an optimal and equitable allocation of COVID-19 vaccines in the Philippines that will minimize the projected number of additional COVID-19 deaths while satisfying the priority groups for immediate vaccination.

Methods In this study, a linear programming model is formulated to determine an allocation of vaccines such that COVID19 deaths are minimized while the prioritization framework set by the government is satisfied. Data used were collected up to November 2020. Total vaccine supply, vaccine effectiveness, vaccine cost, and projected deaths are analyzed. Results of the model are also compared to other allocation approaches.

Results Results of the model show that a vaccine coverage of around $60-70 \%$ of the population can be enough for a community with limited supplies, and an increase in vaccine supply is beneficial if the initial coverage is less than the specified target range. Additionally, among the vaccines considered in the study, the one with $89.9 \%$ effectiveness and a 183 Philippine peso price per dose projected the lowest number of deaths. Compared with other model variations and common allocation approaches, the model has achieved both an optimal and equitable allocation.

Conclusions Having a 100\% coverage for vaccination with a $100 \%$ effectiveness rate of vaccine is ideal for all countries. However, some countries have limited resources. Therefore, the results of our study can be used by policymakers to determine an optimal and equitable distribution of COVID-19 vaccines for a country/community.
\end{abstract}

\section{Introduction}

The coronavirus disease 2019 (COVID-19) is a novel betacoronavirus disease that was initially identified to cause respiratory problems among people from a province in China in late 2019 [1]. It is a viral infection transmitted through respiratory particles, fomites, and other biological matter [2]. Beta-coronaviruses have caused outbreaks in recent decades,

Christian Alvin H. Buhat

chbuhat@up.edu.ph

1 Institute of Mathematical Sciences and Physics, Mathematics Building, University of the Philippines Los Baños, Jose R. Velasco Avenue, Los Baños City 4031, Philippines

2 University of the Philippines Resilience Institute, University of the Philippines Los Baños, Quezon City, Philippines with COVID-19 being characterized as a pandemic by the World Health Organization on the first quarter of 2020 [3, 4].

The Philippines is one of the countries greatly affected by this pandemic [5]. Its government placed the country under community lockdown since March 2020 as a measure to control the spread of the virus [6]. The continuous lockdown contributed to an increase in unemployment rate $(10.4 \%$ as of December 2020) [7], a decrease in overseas remittance inflow (14-20\%) [8], and a decrease in gross domestic product $(9.5 \%$ decline in 2020) [9]. Bringing back jobs is crucial for a sustainable recovery of the economy [10]. This is possible once a sufficiently large proportion of the population becomes immune to the virus and the susceptible population is reduced, leading to what is called herd immunity [11, 12]

There are two general approaches in achieving herd immunity: one is through mass vaccination programs, the 


\section{Key Points for Decision Makers}

Vaccinating around $60-70 \%$ of the population can be beneficial for a resource-constrained country such as the Philippines.

Using the vaccine with an $89.9 \%$ effectiveness rate and a cost of 183 Philippine peso per dose results in a lower number of projected COVID-19 deaths after the rollout of vaccines compared with other vaccines considered in the study.

Following the vaccine distribution from our model results will generate a lower number of projected COVID-19 deaths compared with traditional allocation approaches such as equal allocation, and allocations based on proportion to population size, population density, or the number of COVID-19 cases.

other is through natural immunization of the global population over time [11]. For influenza and following the latter approach, herd immunity was attained after two to three waves where each wave involved seasonal interruptions, immunity from previous flu viruses, or both [12]. For COVID-19, this approach involves costly social and ethical ramifications $[11,12]$. This will require more healthcare resources for patient management and heightened nonpharmaceutical interventions $[11,12]$. Mass vaccination programs are considered to be effective in preventing the spread of the virus and deaths due to infectious diseases such as COVID-19 [12-14]. Vaccination not only reduces infection among the vaccinated, but it also indirectly protects susceptible individuals from secondary infections by reducing the efficiency of virus transmission [15]. It is also seen as the safest means to reach herd immunity [12].

Vaccine development usually takes years to complete from clinical trials to manufacturing [16]. Vaccinating huge groups is another problem entirely. Vaccines have specific storage and shipment requirements to maintain their effectiveness [17]. Once vaccines for COVID-19 are available, healthcare workers should be vaccinated first followed by the elderly and people with pre-existing conditions [12, 18]. This systematic approach would help prevent additional deaths [12]. The Government of the Philippines is following the same prioritization but with the addition of indigenous people and uniformed personnel in the list [19]. The plan is also to prioritize those from the cities to expedite the recovery of the local economy [20]. Distribution of vaccines will be performed through local government units. Because the vaccines are limited by budget, proper allocation of doses for each local government units is vital. An optimal allocation can be found by examining the problem as a constrained optimization (CO) problem that minimizes the number of deaths and follows the prioritization approved by the government.

Constrained optimization is a method of optimizing a set of decision variables. This set of decision variables is often represented by a mathematical equation called the objective function. The optimal values of the decision variables can be determined by subjecting the objective function to additional constraints. In modeling real-world problems, these constraints may include financial, logistical, and human resources. Constrained optimization has been used in managing healthcare responses to existing and emerging threats. It can be used to find the best method to distribute vital resources and determine which issue should receive immediate attention. In 2019, Olbrecht et al. [21] used CO to rank vaccination schemes that control the incidence of pneumococcal diseases among children and the elderly in Brazil. Because of the availability of several vaccines for these diseases, the research aimed to assess the best method of vaccination that complies with the financial constraints and maximizes the quality-adjusted life-year gains in a 10 -year projection. As an effort to determine which public health issue must be prioritized in Malaysia, Varghese et al. [22] developed a CO model for infectious diseases. This model identified pneumococcal disease, dengue, hepatitis $\mathrm{B}$ and $\mathrm{C}$, rotavirus, neonatal pertussis, and cholera as important public health concerns. By considering the prophylactic and clinical treatments for these diseases, the results showed that rotaviral and pneumococcal vaccines take precedence when time horizons are ignored. If the goal is to reduce the number of cases in a 20-year horizon, the results indicated that dengue vaccination must be prioritized. In an age where the spread of a contagious and life-threatening infection is an ever-present risk, these examples demonstrate the practicality of $\mathrm{CO}$ in formulating policies that curb public health threats. In this article, $\mathrm{CO}$ is performed by using a linear programming model to determine an optimal allocation of vaccines for every city or province in the country.

\section{Methods}

The linear program for the vaccine allocation model in this paper aims to minimize COVID-19 deaths after the vaccine rollout while satisfying the prioritization of certain groups for vaccination as identified by the government. The formulation of the objective function and constraints are discussed in the succeeding sections. Throughout the paper, we denote the city or province where the individuals are residing as locality $i$. 


\subsection{Objective Function Formulation}

The objective function value is interpreted as the projected additional COVID-19 deaths after the rollout of vaccines. It is formulated as a summation of multiplicative expressions involving the following parameters.

\subsubsection{Estimation of the Number of Susceptible Individuals After Vaccination}

To estimate the number of susceptible individuals after vaccination rollout in each locality $i$, the population size $N_{i}$, the total current COVID-19 cases $C_{i}$, the number of individuals for vaccination $m_{i}$, and the effectiveness rate of the vaccine eff are needed. The estimate is given by:

$N_{i}-C_{i}-\mathrm{eff} \times m_{i}$,

the model, $N_{i}$ is based on the 2020 population forecast of the 2015 Philippine Statistics Authority Census [23] while $C_{i}$ is the total COVID-19 cases in the Philippines (as of 10 November, 2020) from the Department of Health Data Drop [24]. For the decision variable $m_{i}$, individuals who have already been previously infected are assumed to be either expired cases or recovered cases. For the recovered cases, they obtain a certain degree of immunity to COVID-19 [25]. This suggests that infected individuals can be excluded from the individuals for vaccination, which should be less than $N_{i}-C_{i}$, to further stretch the distribution of vaccines in many localities [26]. Including $C_{i}$ in the objective function does not mean that recovered cases cannot be immunized in the actual implementation of vaccination plans. Recovered cases can be vaccinated depending on the date of recovery, risk of exposure, and the prioritization of the government.

\subsubsection{Determination of Maximum Outbreak Size}

The maximum outbreak size [27] is computed by multiplying the number of individuals susceptible to the disease $N_{i}-C_{i}-$ eff $\times m_{i}$ to:

$1-\frac{1+\log \left(R_{0 i}\right)}{R_{0 i}}$

where $R_{0 i}$ is the maximum reproduction number recorded per locality $i$.

In the model, the maximum reproduction number recorded per locality $i$ as of 11 November, 2020 [28] is used. Note that some localities recorded a very high maximum reproduction number despite their small number of cases because of their small populations [29]. Thus, we set $\max \left\{R_{0 i}\right\}=4$ to be the maximum reproduction number to be considered in this study [30].

\subsubsection{Inclusion of Population Density}

A scaling contact coefficient described as

$1-\exp \left(-\frac{\delta_{i}}{\max \left\{\delta_{i}\right\}}\right)$,

is included to incorporate the contact rate among the members of the population, which is affected by the nonpharmaceutical interventions (e.g., social distancing), and population density $\delta_{i}$ of each locality $i$ [31]. In the Philippines, the population density and the number of cases per province/region are highly correlated. The scaling contact coefficient gives the probability of contact in the population, which is nonlinearly proportional to the population density in that locality. This coefficient characterizes the heterogeneous distribution of cases in a spatial scale, which relaxes the well-mixing (homogeneity) assumption in the epidemiological model $[32,33]$. This heterogeneity in infected cases, as well as deaths, can be observed in the Philippine setting as shown in our data dashboard (https://datastudio. google.com/s/uCv8ZX2rI8w). For the Philippines, we have included the scaling contact coefficient in the optimization model, but for other countries, this coefficient may not be included or may be modified depending on the nature of the COVID-19 dynamics.

\subsection{Constraints Formulation}

The following are parameters needed for the constraints of the linear programming model.

\subsubsection{Prioritization of Certain Groups}

Because of the initial limited amount of vaccines that the country can obtain, priority groups were set by the Government of the Philippines. These include the frontline health workers and uniformed personnel, owing to their high risk of exposure while on duty, and the senior citizens and indigent population because of their vulnerability and by the principle of equity [26, 34]. Moreover, according to [35], when there is a very limited vaccine availability, the priority groups in the setting 'community transmission' are the health workers and the older adults. This was prepared by the SAGE Working Group on COVID-19 vaccines. They defined 'community transmission' as countries or territories experiencing larger outbreaks of local transmission. In the USA, the Interim Framework for COVID Vaccine Allocation of Johns Hopkins University included those at greatest risk of severe illness and death in priority groups for vaccine allocation. These groups were based on their multidisciplinary team's analysis on August 2020 [36]. In a study by [37], 
overwhelmed healthcare facilities (often because of atrisk individuals getting infected) induce lockdowns, which then affects the livelihood of many individuals. Thus, having an equitable distribution of vaccines to these priority groups is necessary for the gradual opening of the economy [38].

To cater to the priority groups set by the government, $G_{i}$ is set to be the number of individuals belonging to the priority group per locality $i$ [24]. This is set to be the minimum number of individuals for vaccination per locality.

\subsubsection{Incorporation of Vaccination Cost}

A crucial factor for resource-constrained countries is the vaccination expense [39]. Some countries are interested in ac quiring multiple vaccines. Total expenses should be less than or equal to the allotted budget $B$. In the Philippines, the approved national budget for vaccines is 72.5 billion Philippine pesos (Php) [40], which will cover vaccine dosage costs as well as the cost of training per vaccinator (1200 Php) and the cost of other peripherals such as masks, face shield, alcohol, and cotton balls (1924 Php for two doses), both of which are good for 350 people [41]. The total vaccination cost is defined as

$\sum_{i}\left(P m_{i}+\frac{m_{i}}{350} \times(1200+1924)\right)$,

where $P$ is the price per complete dose of the COVID-19 vaccine.

\subsection{Linear Programming Model}

The linear programming model formulated to determine the optimal distribution of vaccines among localities in the Philippines is described below. across the country. This model aims to find an optimal allocation that minimizes the projected number of additional COVID-19 deaths in each locality $i$, such that (i) the total number of vaccines for allocation in each locality $i$ will not exceed the total number of available vaccines, (ii) the individuals for vaccination per locality $i$ will not include the number of individuals who have already been infected, (iii) all members of the priority groups in each locality $i$ will be vaccinated, and (iv) the total cost of vaccines that will be used will not exceed the budget allocation. Furthermore, our model is applicable for a long period because of the stable trend of infected individuals and its correlation to the population density.

Constrained optimization was used to compute for the optimal allocation under different approaches that minimize COVID-19 deaths while satisfying the prioritization set by the government. Sensitivity analysis, effects of changes in levels of vaccine supply, vaccine effectiveness, and costs of vaccine were also studied.

\section{Results}

\subsection{Optimal Vaccine Allocation and Sensitivity Analysis}

Budget is a huge consideration in a vaccination program, thus it is only fitting to study the effects of budget in the distribution of the vaccines. The models where budget is considered (limited supplies and budget) and when it is not (limited supplies only) are explored in this section. The "limited supplies" model is the basis for Sect. 3.2. For both approaches, it is assumed that the total number of vaccines for allocation is equal to $50 \%$ of the total population and that the effectiveness rate of the vaccine is $90 \%$, with $2379 \mathrm{Php}$

Minimize $\sum_{i}\left(\left(N_{i}-C_{i}-\operatorname{eff} \times m_{i}\right)\left(1-\frac{1+\log \left(R_{0 i}\right)}{R_{0 i}}\right) \times\left(1-\exp \left(-\frac{\delta_{i}}{\max \left\{\delta_{i}\right\}}\right)\right)\right) d_{i}$

subject to

$$
\begin{aligned}
& \sum_{i} m_{i} \leq V \\
& m_{i} \leq N_{i}-C_{i} \\
& m_{i} \geq G_{i} \\
& \sum_{i}\left(P \times m_{i}+\frac{m_{i}}{350}(1200+1924)\right) \leq B .
\end{aligned}
$$

where $d_{i}$ is the COVID-19 fatality rate per locality $i$ and $V$ is the total available complete doses of vaccine for allocation as its cost per complete dose (similar to a market-available vaccine of $90 \%$ efficacy).

Results show that the total number of complete vaccinations to be distributed across the country is $30,361,078$ when the budget is considered and 54,973,950 when it is not, with corresponding deaths of 17,053 and 6795 , respectively. This translates to an average of 562 and 123 deaths per million vaccines, respectively. The more realistic scenario where budget and supplies are limited is shown in Fig. 1b, d. This case has a lower vaccine allocation ergo more deaths, hence, supporting the need for a greater budget to achieve lower mortality. Furthermore, notice from Fig. 1 that localities 
with the greater share of vaccines still resulted in large projected deaths. This can be explained by the population density and fatality risk in the locality that drive the increase in projected deaths.

Sensitivity analysis on the vaccine supply shows that ceteris paribus, for every $1,000,000$ units of increase in vaccine supply, deaths will only decrease by 112 . If additional vaccines will be acquired, the best option for every unit of increase in supply would be to allocate it to Manila because for every 1000 additional vaccine allocations in Manila, projected deaths will decrease by around 5.

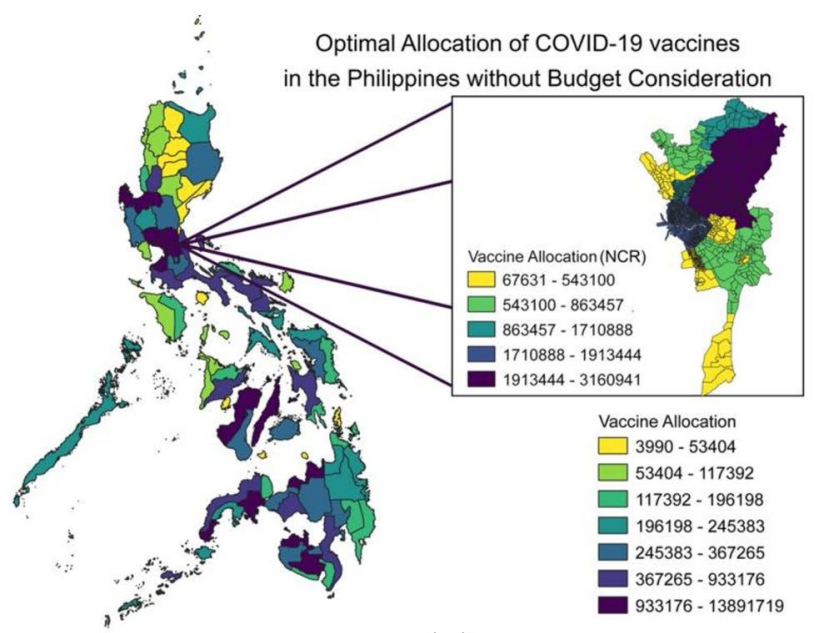

(a)

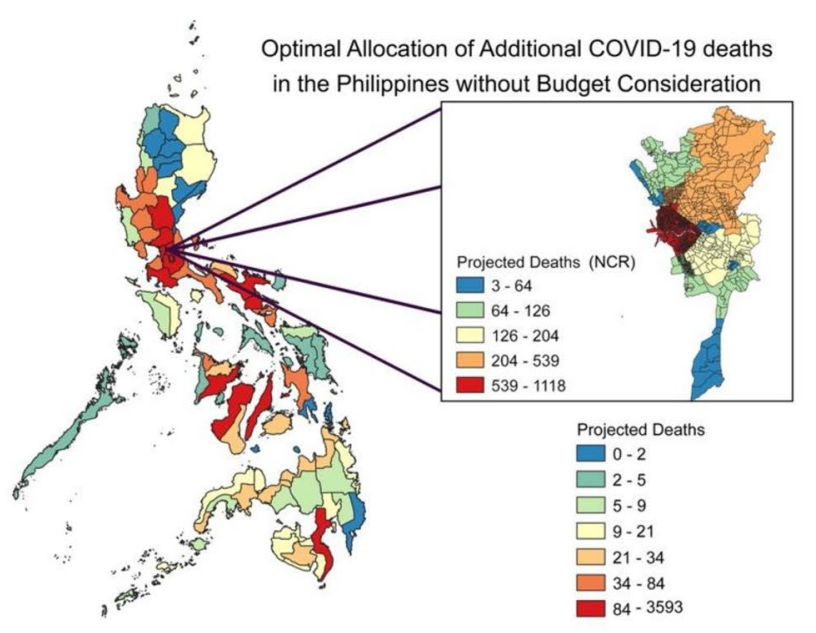

(c)

Fig. 1 Geographical heat maps of vaccine allocations (a without budget consideration and $\mathbf{b}$ with budget consideration) and their corresponding additional deaths (c without budget consideration and $\mathbf{d}$ with budget consideration) with at most $50 \%$ of the national popula-

\subsection{Parameter Analysis and Other Model Cases}

Total vaccine supply, vaccine effectiveness, vaccine cost, and projected deaths are seen as important factors that influence vaccine allocation. Pairwise comparison of different levels of these factors is presented. Other approaches in allocating the vaccine are also studied and discussed below.

\subsubsection{Vaccine Effectiveness and Total Vaccine Supply Levels Comparison}

The total vaccines to be allocated vary from 20 to $100 \%$ of the total population, and the effectiveness rate of vaccines from 50 to $100 \%$ in the linear programming model without

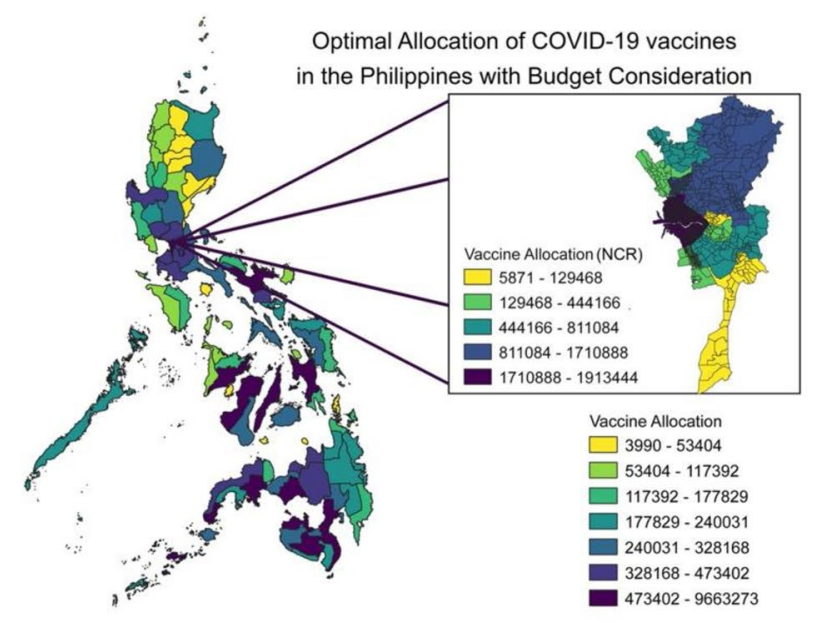

(b)

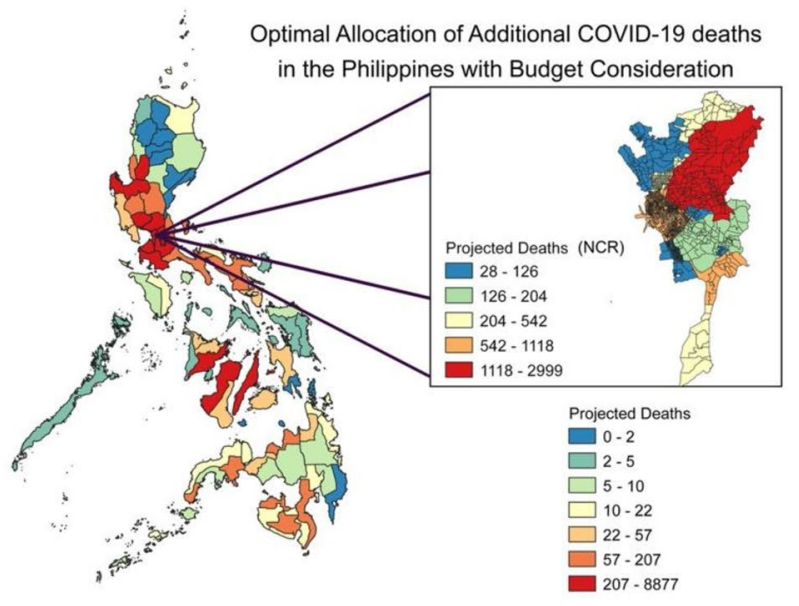

(d)

tion vaccinated, and with a $90 \%$ vaccine effectiveness rate. Details of the distribution per locality can be found in the Electronic Supplementary Material. NCR National Capital Region 
budget consideration. Note that $20 \%$ is assumed in the model to be the minimum total vaccines for allocation to accommodate all priority groups [34], and 50\% to satisfy the minimum efficacy rate for COVID-19 vaccines [42].

Figure 2 shows that the greater the number of people for vaccination coupled with a high vaccine effectiveness rate, the fewer number of additional deaths that might occur. This is an expected result. The ideal set-up in a nationwide vaccination program is to maximize both factors. Unfortunately, not all countries have the resources to provide $100 \%$ coverage using the vaccine with the highest effectiveness rate. Achieving herd immunity can be the minimum goal instead. However, in the heat map, notice the blue and red regions. Assume that the blue squares represent the low projected deaths and the red squares represent the high projected deaths. With a low coverage of vaccination, even if there is $100 \%$ effectiveness, it will result in a red square (high number of cases). However, as the coverage increases, the less

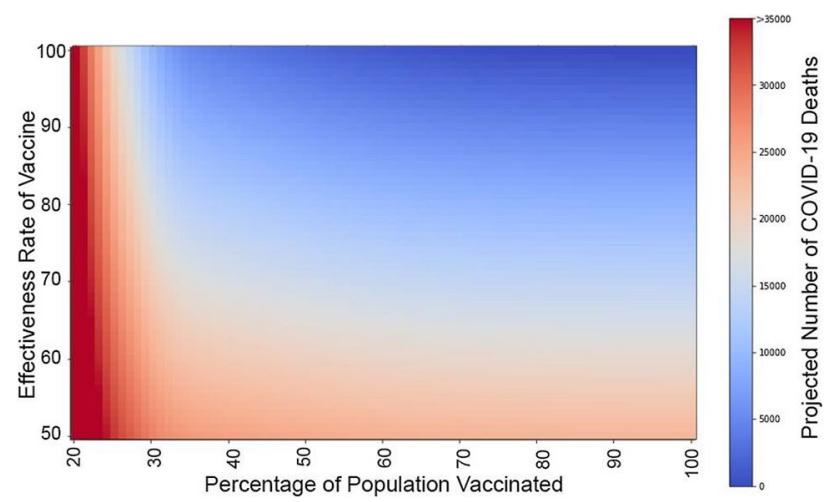

Fig. 2 Heat maps of the resulting objective function values (projected additional deaths) from the linear programming model without budget consideration as the percentage of the population to be vaccinated varied from 20 to $100 \%$ and the vaccine effectiveness varied from 50 to $100 \%$ minimum effectiveness necessary to achieve a blue square (low number of cases). In fact, according to the results of Salaverria et al. [43], to stop an ongoing epidemic, it is recommended that the minimum efficacy of the vaccine should be $60 \%$ when coverage is $100 \%$, and at least $80 \%$ when coverage falls to $75 \%$, to reduce the peak of the epidemic. Both cases fall under the blue region, among other efficacy-coverage combinations, which are feasible in the Philippines because protection and interventions will still be implemented even after vaccination [44].

We further investigate the behavior of the percentage of the vaccinated population and vaccine effectiveness as they increase. From Fig. 3a, the projected deaths slowed down at after $20-40 \%$ and at around $60-70 \%$, the effect of increasing in the percentage of population vaccinated is minimal. This explains the low decrease in deaths from Fig. 2, despite the huge increase in units of vaccine supply. A reason for this is because $60-70 \%$ is near the $1-\frac{1}{R_{0}}$ threshold for immunization coverage of the $\mathrm{R} 0$ country, assuming that $R_{0}$ is at a maximum [45]. This result is validated by the recommendation of Gavi the Vaccine Alliance [46] that at least $60 \%$ of the population should be immunized to achieve herd immunity. While the actual proportion of vaccination to achieve herd immunity against COVID-19 is not known [47], our result makes for a good target for countries with insufficient resources to vaccinate all of its constituents. If the budget is tight, to accommodate constrained resources, inoculating at least $50 \%$ of the population would still reduce the projected COVID-19 deaths but it might not result in herd immunity.

Meanwhile, in Fig. 3b, the vaccine effectiveness increases have been shown to have a linear relationship with the projected deaths. That is, a vaccine with a higher effectiveness rate always generates lower deaths. Vaccine effectiveness rates should be maximized as much as possible when deciding on what vaccine should be procured. Furthermore, notice the change in slope as the objective function value decreases,
Fig. 3 Projected additional death from the linear programming model as the percentage of population for vaccination and vaccine effectiveness are varied

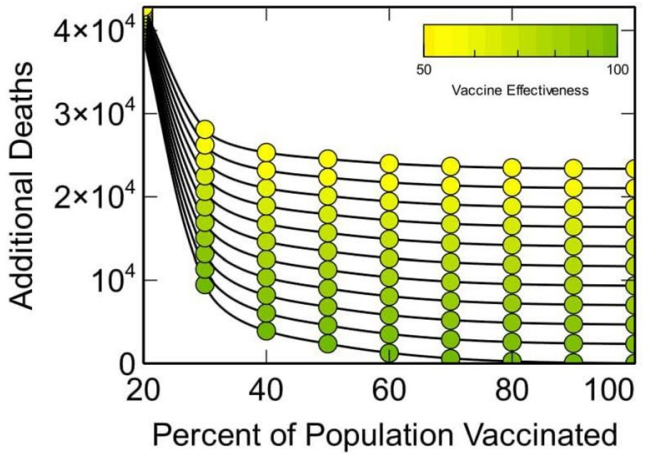

(a)

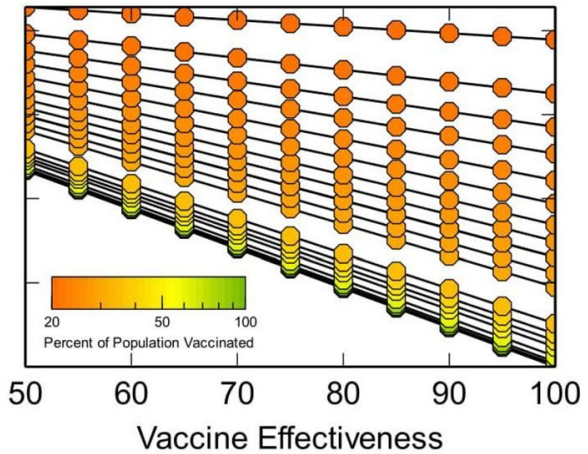

(b) 
which implies that the higher the objective function value (additional deaths), the lesser the effect of increasing the effectiveness of the vaccine, which is derivative from the result from Fig. 2 where the blue region gets larger as the number of population to be vaccinated increases and the number of deaths decreases.

\subsubsection{Cost Analysis in Vaccine Acquisition}

Another key factor in determining what vaccine to use is the cost of the vaccine. Each vaccine has its price per dose, required vaccine dose per person, and efficacy rate (also known as the effectiveness rate). Values of which are based on the market-available vaccines in the Philippines. These factors are used for comparative analysis of the vaccines.

Among the vaccines, vaccines 1,2 , and 7 can provide $100 \%$ national coverage, i.e., vaccinate all 110 million plus residents of the country. All but vaccine 4 can fulfill the prioritization of certain groups identified by the government. Vaccine 2 generated the lowest projected number of additional deaths compared with the other vaccines. This is because of its high effectiveness rate and significantly low price per dose.

Vaccines 3 and 4 can only cover $28 \%$ and $16 \%$ of the population, respectively, and have high death projections despite having high vaccine effectiveness because of its high cost per dose. Thus, a significant increase in the total budget for vaccination will drastically decrease their additional death projections (see Fig. 4).

\subsubsection{Exploring Other Approaches in Vaccine Allocation}

Multiple countries use different approaches in distributing vaccines in their community. Table 1 shows the other approaches for vaccine allocation that can also be adapted, including the one presented in Sect. 3.1.

The first approach that considers the allocated budget for vaccines presents the likely scenario in the Philippine setting, while the remaining approaches (without a budget constraint) can be viewed as approaches that have an equal budget allotment and are thus comparable without a budget constraint. The first two cases in Table 1 are those presented in Sect. 3.1. The third approach assumes that the $R_{0}$ in every locality $i$ is 4 (maximum) to further estimate what to expect if $\mathrm{R} 0$ gets bigger. As expected, there are greater projected deaths even with slight changes in the allocation per locality. If there is no prioritization of certain groups of individuals, the lower bound in constraint 3 of the model (see Sect. 2.3) is removed. This is the fourth approach that resulted in even fewer deaths. However, this approach is inequitable as many localities will not receive vaccines.

The next three approaches follow allocations based on proportionality, i.e., if a country will allocate the vaccine based on the population size of the locality, then the vaccine allocation will be proportional to the population of that locality. Note that allocations per locality cannot exceed the $\mathrm{Ni}-\mathrm{Ci}$ susceptible population of that province. Allocating vaccines based on population size resulted in the most number of death projections while allocating based on the number of infections generated the least. None among the three proportion allocations had better results compared

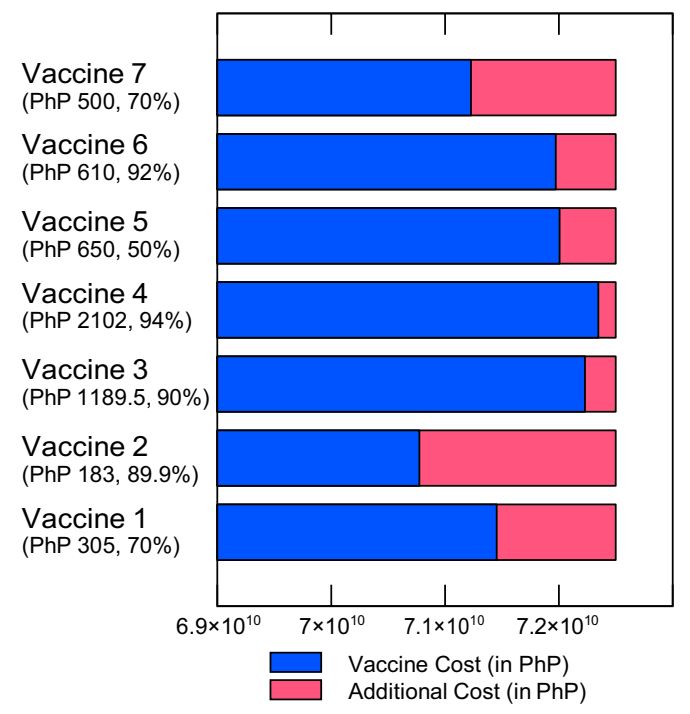

Fig. 4 Multiple vaccines and their effectiveness (percentage inside the brackets), cost in Philippine pesos (Php) [1 Php = US\$0.021] of vaccine (cost inside the brackets) and additional costs (training per

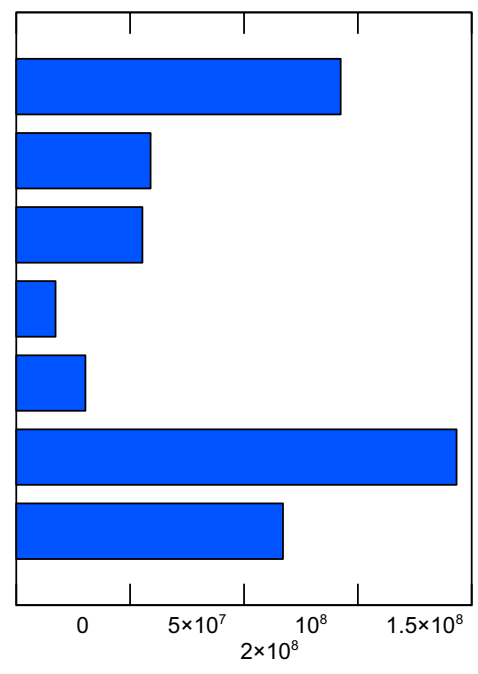

Feasible Recipients

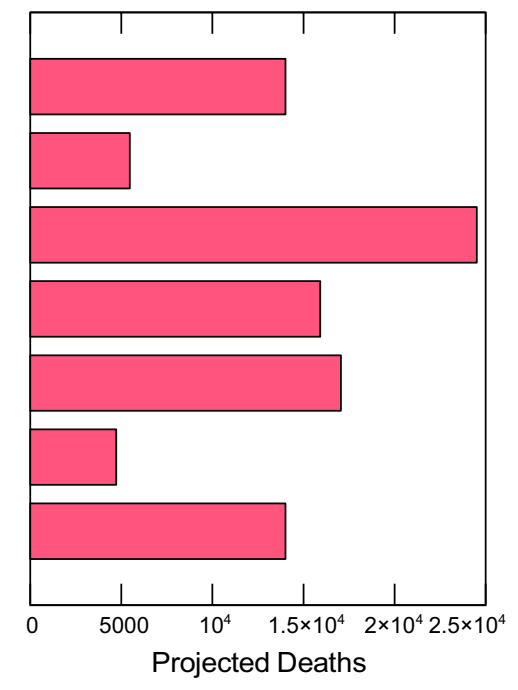

vaccinator, and other peripherals such as masks, face shield, alcohol, and cotton balls), and projected number of additional deaths based from our model 
Table 1 Different approaches to vaccine allocation and its projected number of deaths. The last four rows are without budget consideration

\begin{tabular}{llc}
\hline Vaccine allocation approaches & $\begin{array}{l}\text { Total number of complete } \\
\text { vaccinations }\end{array}$ & $\begin{array}{l}\text { Projected addi- } \\
\text { tional number of } \\
\text { deaths }\end{array}$ \\
\hline Linear programming model result WITH budget consideration & $30,361,078$ & 17,053 \\
Linear programming model result WITHOUT budget consideration & $54,973,950$ & 6795 \\
Linear programming model result WITHOUT budget consideration and with $R_{0}=4$ & $54,973,950$ & 8482 \\
Linear programming model result WITHOUT budget consideration and WITHOUT lower & $54,973,950$ & 5900 \\
$\quad$ bound & & 25,457 \\
Allocation proportional to population size & $54,973,949$ & 12,588 \\
Allocation proportional to population density & $19,952,176$ & 8433 \\
Allocation proportional to number of infections & $42,391,915$ & 25,955 \\
Equal allocation & $54,973,950$ & \\
\hline
\end{tabular}

with the linear programming model result without a budget consideration.

The last approach assumes equal allocation among localities regardless of factors considered in the previous three approaches. This, in turn, results in the most number of additional deaths, and thus, is the least ideal method to allocate the vaccines as it does not consider the variability in fatality rates, infections, and dynamics in each locality. Comparing the "without budget" constrained approaches, the linear programming model results in the second approach are the best in terms of minimizing the number of deaths and following equitable considerations.

\section{Discussion}

COVID-19 vaccine allocation is an immediate concern globally as it is perceived as the solution to control the pandemic by achieving herd immunity in every country. A linear programming model is used to study vaccine allocation. This $\mathrm{CO}$ approach aims to minimize deaths while satisfying group prioritization for immediate vaccination. Various approaches were studied, all of which assumed using a vaccine with a $90 \%$ effectiveness rate that is to be administered to at most $50 \%$ of the population.

Sensitivity analysis shows that if only the vaccine supply is limited and budget is not a constraint, deaths will decrease by 112 for every 1 million vaccines. As seen in Sect. 3.2.1, when vaccine coverage is at around $60-70 \%$, which is the case for the non-budget constrained approach, a decrease in deaths will not be as apparent as when coverage is less than the specified range. Therefore, additional vaccines beyond the optimal allocation will be beneficial in decreasing deaths only if the vaccine coverage is less than $60 \%$.

Various vaccines were also studied in terms of their associated costs and effectiveness, and it was determined that the vaccine with $89.9 \%$ effectiveness and 183 Php price per dose resulted in the lowest projected deaths. We then compared our result with other model variations and common allocation approaches, upon which our model achieved both the optimal and a more equitable allocation.

Among the approaches that countries can adopt, the approach using our vaccine allocation model projects the least amount of deaths while upholding equitable distribution. Although the proportional allocation according to the number of infections can be achieved more conveniently, this allocation will not yield minimal deaths. Our model, thus, can be used by policymakers as the minimization of casualties is a humane priority. Furthermore, our model provides insight into the localities that should be prioritized when additional vaccine doses become available. The framework that we used for the equitable allocation of the vaccine that prioritizes the older age groups was also used by the panel of experts from the Centers for Disease Control and Prevention and the National Institutes of Health [48, 49]. Our findings follow from the results of Foy et al. [50] and Tuite et al. [51], which suggest that vaccines should be allocated to high-risk groups to reduce morbidity and mortality.

Using our model can help policymakers in allocating COVID-19 vaccines, especially if their resources are limited. This model is applicable for a long period owing to the stable trend of infected individuals and its correlation to the population density. Furthermore, it can be applied and translated to other resource-constrained countries around the world. The number of COVID-19 cases and deaths, fatality risks, distribution channels, budget, vaccine effectiveness, the minimum number of population to be vaccinated, and other key inputs can be easily adjusted in our model to adapt to the needs of each country. By having an optimal and equitable distribution of vaccines, resources are well utilized and not wasted. With sufficient data, the model can also be used for other similar infectious diseases and can be used as a basis in future vaccination programs. 


\section{Limitations}

Our model is limited to the use of one brand of vaccine. Incorporating multiple vaccines all at once can be part of future research work. Additionally, the model assumes that the government will distribute the vaccine, and thus vaccinations acquired from companies or individuals were not considered. Incorporation of other factors such as new COVID19 strains, dynamic herd immunity, group-specific infection rates, clinical trial results of vaccines, differential vaccine transportation costs, and storage facilities, among other factors also provide research directions that warrant attention.

Other countries can use different parameters of the optimization model, such as the number of recovered cases and the effect of population density (scaling contact coefficient) in the objective function. The decision of whether to include such parameters depends on the set prioritization plans by the government, and on the tolerance to exclude heterogeneity in COVID-19 transmission. The CO model assumes the maximum outbreak size, which is static. The $\mathrm{CO}$ model can be coupled with a dynamic system model to reflect the speed and efficiency of implementing temporal vaccination plans.

Supplementary Information The online version contains supplementary material available at https://doi.org/10.1007/s40258-021-00667-z.

Acknowledgments Jomar F. Rabajantea is supported by the Abdus Salam International Centre for Theoretical Physics Associateship Scheme.

\section{Declarations}

Funding This research is funded by the UP System through the UP Resilience Institute.

Conflicts of interest/Competing interests Christian Alvin H. Buhat, Destiny SM. Lutero, Yancee H. Olave, Kemuel M. Quindala III, Mary Grace P. Recreo, Dylan Antonio SJ. Talabis, Monica C. Torres, Jerrold M. Tubay, and Jomar F. Rabajante have no conflicts of interest that are directly relevant to the content of this article.

Ethics approval Not applicable.

Consent to participate Not applicable.

Consent for publication Not applicable.

Availability of data and material The data per locality and linear programming computations can be found online at: http://github.com/alvin izer/vaccineallocation/.

Code availability The Microsoft Excel and Python file used for the model simulation can be found online at: http://github.com/alvinizer/ vaccineallocation/.

Authors' contributions All authors were involved in the drafting and revising of the manuscript.

\section{References}

1. World Health Organization. Coronavirus disease (COVID-19) situation report 1 Philippines 9 March 2020. 2020. https://www. who.int/docs/default-source/wpro-documents/countries/phili ppines/emergencies/covid-19/who-phl-sitrep-1-covid-19-9mar2 020.pdf?sfvrsn=2553985a_2. Accessed 17 Dec 2020.

2. World Health Organization. Transmission of SARS-CoV-2: implications for infection prevention precautions. 2020. https:// www.who.int/news-room/commentaries/detail/transmission-ofsars-cov-2-implications-for-infection-prevention-precautions. Accessed 17 Dec 2020.

3. Kaur SP, Gupta V. COVID-19 vaccine: a comprehensive status report. Virus Res. 2020. https://doi.org/10.1016/j.virusres.2020. 198114.

4. World Health Organization. WHO Director-General's opening remarks at the media briefing on COVID-19: 11 March 2020. 2020. https://www.who.int/director-general/speeches/detail/ who-director-general-s-opening-remarks-at-the-media-briefingon-covid-1911-march-2020. Accessed 17 Dec 2020.

5. Malindog-Uy A. COVID-19 impacts in The Philippines. 2020. https://theaseanpost.com/article/covid-19-impacts-philippines. Accessed 15 Jan 2021.

6. Buhat CA, Villanueva SK. Determining the effectiveness of practicing non-pharmaceutical interventions in improving virus control in a pandemic using agent-based modelling. Math Appl Sci Eng. 2020. https://doi.org/10.5206/mase/10876.

7. The Straits Times. Philippines suffers worst job losses in 15 years due to Covid-19 and lockdown. 2020. https://www.straitstimes. com/asia/se-asia/philippines-suffers-worst-job-losses-in-15-yearsdue-to-covid-19-and-lockdown. Accessed 15 Jan 2021.

8. Murakami E, Shimizutani S, Yamada E. Projection of the effects of the COVID-19 pandemic on the welfare of remittance-dependent households in the Philippines. EconDisCliCha. 2020. https:// doi.org/10.1007/s41885-020-00078-9.

9. Philippine Statistics Authority. Philippine GDP posts -8.3 percent in the fourth Quarter 2020; -9.5 percent for full-year 2020. 2021. https://psa.gov.ph/content/philippine-gdp-posts-83-percentfourth-quarter-2020-95-percent-full-year-2020. Accessed 8 Feb 2021.

10. de Vera B. COVID-19 impact: IMF sees PH GDP dropping by record 8.3 percent in 2020. 2020. Available from: https://busin ess.inquirer.net/309494/covid-19-impact-imf-sees-ph-gdp-dropp ing-by-record-8-3-percent?fbclid=IwAR3mdzuouQZVNYwEMS wdSYA4eXn4TIcade5cwJGFcAa. Accessed 15 Jan 2021.

11. Herd immunity: understanding COVID-19. Immunity. 2020;52.5:737-41. https://doi.org/10.1016/j.immuni.2020.04.012.

12. Fontanet A, Cauchemez S. COVID-19 herd immunity: where are we? Nat Rev Immunol. 2020. https://doi.org/10.1038/ s41577-020-00451-5.

13. World Health Organization. International travel and health. Geneva: World Health Organization; 2020.

14. Toumi M, Ricciardi W. The economic value of vaccination: why prevention is wealth. J Mark Access Health Policy. 2015. https:// doi.org/10.3402/jmahp.v3.29414.

15. John TJ, Samuel R. Herd immunity and herd effect: new insights and definitions. Eur J Epidemiol. 2000;16(7):601-6. https://doi. org/10.1023/a:1007626510002.

16. Centers for Disease Control and Prevention. Vaccine testing and the approval process. 2014. https://www.cdc.gov/vaccines/basics/ test-approve.html. Accessed 15 Jan 2021.

17. World Health Organization. Manufacturing, safety and quality control of vaccines. 2020. https://www.who.int/news-room/ feature-stories/detail/manufacturing-safety-and-quality-control. Accessed 15 Jan 2021. 
18. World Health Organization. Science in 5 on Covid-19: episode 5. 2020. https://www.who.int/emergencies/diseases/novel-coron avirus-2019/media-resources/science-in-5/episode-5. Accessed 15 Jan 2021.

19. CNN Philippines. PH not yet ready to handle Pfizer COVID-19 vaccine doses by January - Romualdez. 2020. https://cnnphilipp ines.om/news/2020/12/21/PH-not-yet-ready-to-handle-PfizerCOVID-19-vaccine-doses-by-January-Romualdez.html. Accessed 15 Jan 2021.

20. Gulla V. Philippines expects to roll out COVID-19 vaccines first half of 2021. 2020. https://news.abs-cbn.com/spotlight/12/10/20/ philippines-expects-to-roll-out-covid-19-vaccines-first-half-of2021? fbclid=IwAR36-JN09LR31tspSd0nP8Wzw81PnEBsjvC z9i4k72-sh739_0. Accessed 15 Jan 2021.

21. Olbrecht J, Gomez J, Van Bellinghen LA, et al. PMU20 constrained optimization for identifying the combination of pneumococcal vaccination schemes which maximizes QALY-gains over the next 10 years in Brazil. Value Health. 2019. https://doi.org/ 10.1016/j.jval.2019.04.1183.

22. Varghese L, Puteh S, Schecroun N, et al. Applying a constrained optimization portfolio model to aid prioritization of public health interventions in Malaysia. Value Health Reg Issues. 2020;21:17280. https://doi.org/10.1016/j.jval.2019.04.1183.

23. Philippine Statistics Authority. Philippine population density (cased on the 2015 Census of Population). 2020.https://psa.gov. $\mathrm{ph} /$ content/philippine-population-density-based-2015-censuspopulation. Accessed 11 Apr 2020.

24. Department of Health. 2020. https://doh.gov.ph/covid19tracker. Accessed 25 Nov 2020.

25. World Health Organization. COVID-19: immunity after recovery from COVID-19. 2020. https://www.who.int/emergencies/ diseases/novel-coronavirus-2019/media-resources/science-in-5/ episode-18-covid-19-immunity-after- recovery-from-covid-19. Accessed 11 Feb 2020.

26. Bubar K, Reinholt K, Kissler S, et al. Model-informed COVID-19 vaccine prioritization strategies by age and serostatus. Science. 2021. https://doi.org/10.1126/science.abe6959.

27. Hartfield M, Alizon S. Introducing the outbreak threshold in epidemiology. PLoS Pathog. 2013;9: e1003277. https://doi.org/10. 1371/journal.ppat.1003277.

28. University of the Philippines. 2020. https://endcov.ph/statistics/. Accessed 25 Nov 2020.

29. David Adam. A guide to R: the pandemic's misunderstood metric. 2020. https://www.nature.com/articles/d41586-020-02009-w. Accessed 8 Feb 2021.

30. World Health Organization. COVID-19: a global pandemic. What do we know about SARS-CoV-2 and COVID-19? 2020. https://www.who.int/docs/default-source/coronaviruse/riskcomms-updates/update-28-covid-19-what-we-know-may-2020. pdf?sfvrsn=ed6e286c_2. Accessed 8 Feb 2021.

31. Hu H, Nigmatulina $K$, Welkhoff $P$. The scaling of contact rates with population density for the infectious disease models. Math Biosci. 2013. https://doi.org/10.1016/j.mbs.2013.04.013.

32. Buhat CAH, Duero JCC, Felix EFO, et al. Optimal allocation of COVID-19 test kits among accredited testing centers in the Philippines. J Healthc Inf Res. 2020. https://doi.org/10.1007/ s41666-020-00081-5.

33. Sanche $\mathrm{S}$, Lin $\mathrm{Y}, \mathrm{Xu} \mathrm{C}$, et al. High contagiousness and rapid spread of severe acute respiratory syndrome coronavirus 2 . Emerg Infect Dis. 2020;26(7):1470-7.

34. Department of Health. FAQS: vaccines. 2020. https://doh.gov. $\mathrm{ph} /$ faqs/vaccines\#: :text $=$ Frontline $\% 5 \mathrm{C} \% 20$ health $\% 5 \mathrm{C} \% 20$ wor kers\%5C\%2C\%5C\%20senior\%5C\%20citizens,9. Accessed 21 Jan 2020
35. World Health Organization. WHO SAGE Roadmap for prioritizing the use of COVID-19 vaccines in the context of limited supply. 2020. https://www.who.int/publications/i/item/whosage-roadmap-for-prioritizing-uses-of-covid-19-vaccines-inthe-context-of-limited-supply. Accessed 18 May 2021.

36. Johns Hopkins Bloomberg School of Public Health. Interim framework for COVID vaccine allocation and distribution in the United States. 2020. https://www.centerforhealthsecurity.org/ our-work/pubs_archive/pubs-pdfs/2020/200819-vaccine-alloc ation.pdf. Accessed 18 May 2021.

37. Kaye A, Okeagu C, Pham A, et al. Economic impact of COVID19 pandemic on healthcare facilities and systems: international perspectives. Best Pract Res Clin Anaesthesiol. 2020. https:// doi.org/10.1016/j.bpa.2020.11.009.

38. National Economic and Development Authority. NEDA recommends priority sectors for A4 group vaccination. 2020. https:// www.neda.gov.ph/neda-recommends-priority-sectors-for-a4group-vaccination/. Accessed 21 Apr 2021.

39. Chootipongchaivat S, Chantarastapornchit V, Kulpeng W, et al. Vaccination program in a resource-limited setting: a case study in the Philippines. Vaccine. 2016;34:4814-9. https://doi.org/10. 1016/j.vaccine.2016.08.014.

40. Department of Budget and Management. GAA 2021. 2021. https://www.dbm.gov.ph/index.php/budget-documents/2021/ general-appropriations-act-fy-2021. Accessed 11 Feb 2020.

41. Katrina Domingo. Which COVID-19 vaccine is most costfriendly for PH government? 2020. https://news.abs-cbn.com/ news/12/10/ 20/which-covid-19-vaccine-is-most-cost-friendlyfor-ph-government. Accessed 10 Dec 2020.

42. Salaverria LB, Aurelio JM. DOH: $50 \%$ vaccine efficacy is OK. 2020. https://newsinfo.inquirer.net/1376429/doh-50-vaccineefficacy-is-ok. Accessed 27 Dec 2020.

43. Bartsch S, O'Shea K, Ferguson M, et al. Vaccine efficacy needed for a COVID-19 coronavirus vaccine to prevent or stop an epidemic as the sole intervention. Am J Prev Med. 2020;59:493503. https://doi.org/10.1016/j.amepre.2020.06.011.

44. Mandavilli A. Here's why vaccinated people still need to wear a mask. 2020. https://www.nytimes.com/2020/12/08/health/covidvaccine-mask.html. Accessed 3 Feb 2021.

45. Fine $P$, Eames K, Heymann D. Herd immunity: a rough guide. Clin Infect Dis. 2011;52:911-6. https://doi.org/10.1093/cid/cir007.

46. Gavi The Vaccine Alliance. Here's why vaccinated people still need to wear a mask. 2020. https://www.gavi.org/vaccineswork/ what-herd-immunity? Accessed 26 Mar 2020.

47. World Health Organization. Here's why vaccinated people still need to wear a mask. 2020. https://www.who.int/news-room/qa-detail/herd-immunity-lockdowns-and-covid-19? Accessed 31 Dec 2020.

48. Gayle H, Foege W, Brown L, et al. Framework for equitable allocation of COVID-19 vaccine, vol 10. Washington, DC: The National Academy Press, 2020.

49. Foy BH, Wahl B, Mehta K, et al. Comparing COVID-19 vaccine allocation strategies in India: a mathematical modelling study. Int J Infect Dis. 2021. https://doi.org/10.1016/j.ijid.2020.12.075.

50. Tuite AR, Fisman D, Kwong J, et al. Optimal pandemic influenza vaccine allocation strategies for the Canadian population. PLoS ONE. 2010. https://doi.org/10.1371/journal.pone.0010520.

51. Russell FM, Greenwood B. Who should be prioritised for COVID19 vaccination? Hum Vaccin Immunother. 2021;17(5):1317-21. https://doi.org/10.1080/21645515.2020.1827882. 